\title{
Ultrasound-derived three-dimensional print models made ultrasimply
}

\author{
William J. Mauermann, MD, ${ }^{\mathrm{a}}$ and Jane M. Matsumoto, $\mathrm{MD}^{\mathrm{b}}$
}

\author{
From the ${ }^{\mathrm{a}}$ Division of Cardiovascular Anesthesiology, Department of Anesthesiology, and the ${ }^{\mathrm{b}}$ Division of \\ Pediatric Radiology, Department of Radiology, Mayo Clinic, Rochester, Minn. \\ Disclosures: Authors have nothing to disclose with regard to commercial support. \\ Received for publication Sept 12, 2017; accepted for publication Sept 15, 2017; available ahead of print Oct 17, \\ 2017. \\ Address for reprints: William J. Mauermann, MD, Department of Anesthesiology, Mayo Clinic College of \\ Medicine, 200 First St SW, Rochester, MN 55905 (E-mail: Mauermann.William@ mayo.edu). \\ J Thorac Cardiovasc Surg 2018;155:146 \\ $0022-5223 / \$ 36.00$ \\ Copyright $(2) 2017$ by The American Association for Thoracic Surgery \\ https://doi.org/10.1016/j.jtcvs.2017.09.080
}

Since the first 3-dimensional (3D) printing technology was introduced in 1986, there has been an exponential increase in its use in a number of fields, including medicine. With this technology, 3D models of cardiac structures can be used for both patient and trainee education, as well as to plan cardiac interventions (eg, sizing of stents, valves, and neochords to repair mitral regurgitation) and to study complex relationships. The creation of a 3D model first requires acquisition of high-resolution clinical imaging studies that use DICOM (Digital Imaging and Communication in Medicine)-based volumetric data sets. To date, computed tomographic imaging has been the modality of choice for obtaining this imaging data because of the ability to acquire thin-section, gated images. ${ }^{1}$ As an alternative, 3D transesophageal echocardiography (TEE) has potential use in acquiring valuable imaging information to use in creating 3D-printed cardiac models. It is easily available and does not use radiation. Although 3D TEE is not the modality of choice for imaging all cardiac structures, it is likely equivalent or superior to other modalities for some clearly defined applications, such as imaging the mitral valve leaflets. Indeed, it has now been more than 15 years since Binder and colleagues ${ }^{2}$ demonstrated the ability to create accurate $3 \mathrm{D}$ prints of the mitral valve from TEE-derived data sets.

One potential reason for the current lack of enthusiasm for the use of TEE data sets in 3D printing is the difficulty in obtaining the required data points and placing them into a useable DICOM format that allows the accurate segmentation of a cardiac structure that can be 3D printed. Currently, only proprietary software is available for creation of $3 \mathrm{D}$ data sets from TEE images. In addition, storage methods of 3D TEE data are vendor specific. In this issue of the Journal, Hosny and colleagues ${ }^{3}$ report the use of customized software for the exportation of TEE-derived 3D images to a format that is compatible with 3D printing. Hosny and colleagues ${ }^{3}$ have made every assurance to the Journal that this software is, and will remain, freeware, and that they have no financial interest. The software runs entirely within the end-

\section{References} 2000;35:230-7.

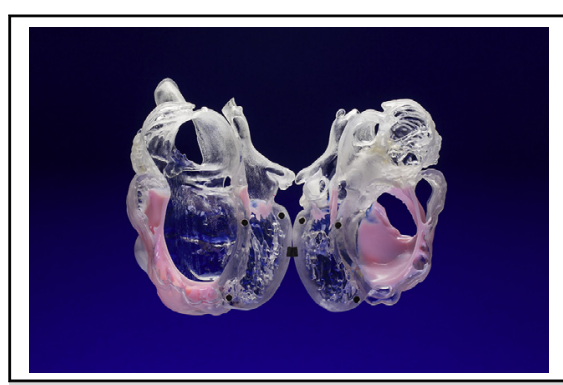

A teaching model, generated with computed tomographic data, showing Ebstein anomaly.

Central Message

This article describes a simplified technique for the extraction of 3-dimensional transesophageal echocardiographic data into a format that allows the printing of a 3-dimensional model.

See Article page 143

user's browser, which obviates concerns regarding dissemination of patient identifiers. The report includes an internet link to a well-executed video that guides the end user step by step through the process of extracting the required data.

Although the software is currently only compatible with files generated from Philips QLab (Philips Healthcare, Andover, Mass), we hope that this group will be able to expand the technology to other vendors. Other limitations include the fact that this software is not US Food and Drug Administration approved, and we currently have no data to support its accuracy. The degree to which a 3D model represents actual patient anatomy is limited to the accuracy of the imaging technology and the software that interprets it. We hope that this group will go forward with further studies to prove the accuracy of their technology.

Clearly, 3D print models have found a place in the medical field, including cardiovascular medicine. Although the software proposed by Hosny and colleagues ${ }^{3}$ may not be the final step, it is certainly a step in the right direction.

1. Vukicevic M, Mosadegh B, Min JK, Little SH. Cardiac 3D printing and its future directions. JACC Cardiovasc Imaging. 2017;10:171-84.

2. Binder TM, Moertl D, Mundigler G, Rehak G, Franke M, Delle-Karth G, et al. Stereolithographic biomodeling to create tangible hard copies of cardiac structures from echocardiographic data: in vitro and in vivo validation. J Am Coll Cardiol.

3. Hosny A, Shen T, Kuo AS, Long D, Andrawes MN, Dilley JD. Unlocking vendorspecific tags: three-dimensional printing of echocardiographic data sets. J Thorac Cardiovasc Surg. 2018;155:143-5.e1. 